Research Paper

\title{
FOLFIRINOX in Locally Advanced and Metastatic Pancreatic Cancer: A Single Centre Cohort Study
}

\author{
SJ Rombouts1,2, TH Mungroop ${ }^{1}$, MN Heilmann³, HW van Laarhoven³, OR Busch¹, IQ Molenaar1,2, \\ MG Besselink ${ }^{1}$, JW Wilmink ${ }^{3} \bowtie$ \\ 1. Department of Surgery, Academic Medical Center Amsterdam, PO Box 22660, 1100 DD Amsterdam, The Netherlands \\ 2. Department of Surgery, University Medical Center Utrecht, PO box 85500, 3508 GA Utrecht, The Netherlands \\ 3. Department of Medical Oncology, Academic Medical Center Amsterdam, PO Box 22660, 1100 DD Amsterdam, The Netherlands \\ $\triangle$ Corresponding author: J.W. Wilmink, MD, PhD, Academic Medical Center Amsterdam, Department of Medical Oncology, G4-212, PO Box 22660, 1100 DD \\ Amsterdam, The Netherlands. E-mail: j.w.wilmink@amc.nl. Tel.: +31 205669111
}

(C) Ivyspring International Publisher. Reproduction is permitted for personal, noncommercial use, provided that the article is in whole, unmodified, and properly cited. See http://ivyspring.com/terms for terms and conditions.

Received: 2016.05.24; Accepted: 2016.07.15; Published: 2016.08.26

\begin{abstract}
Introduction: FOLFIRINOX is emerging as new standard of care for fit patients with locally advanced pancreatic cancer (LAPC) and metastatic pancreatic cancer (MPC). However, some of the physicians are reluctant to use FOLFIRINOX due to high toxicity rates reported in earlier studies. We reviewed our experience with FOLFIRINOX in LAPC and MPC, focussing on dose adjustments, toxicity and efficacy.

Methods: We reviewed all patients with LAPC or MPC treated with FOLFIRINOX in our institution between April 2011 and December 2015. Unresectability (stage III and IV) was determined by the institution's multidisciplinary team for pancreatic cancer.

Results: Fifty patients (18 LAPC and 32 MPC) were enrolled, with a median age of 55 years (IQR 49-66) and WHO performance status of $0 / 1$. FOLFIRINOX was given as first-line treatment in $82 \%$ of patients. Dose modifications were applied in $90 \%$ of patients. The median number of completed cycles was 8 (IQR 5-9). Grade 3-4 toxicity occurred in $52 \%$ and grade 5 toxicity in $2 \%$. The response rate was $25 \%$ (12\% in LAPC, 32\% in MPC). Median overall survival and progression-free survival were 14.8 and 10.3 months in LAPC, and 9.0 and 5.9 months in MPC, respectively. Overall 1- and 2-year survival was $65 \%$ and $10 \%$ in LAPC and $40 \%$ and $5 \%$ in MPC. Within the LAPC group, 6 patients (33\%) underwent local ablative therapy and 1 patient (6\%) a resection, leading to a median survival of 21.8 months.

Conclusion: FOLFIRINOX treatment with nearly routine dose modification was associated with acceptable toxicity rates, relatively high response rates and an encouraging overall survival.
\end{abstract}

Key words: FOLFIRINOX, pancreatic cancer, advanced stage, stage III and IV, toxicity, efficacy

\section{Introduction}

Pancreatic cancer is the 4th leading cause of cancer related death worldwide [1]. In Europe, the incidence of pancreatic cancer is around 103,800 patients [2]. In 2015, the incidence in the Netherlands was around 2,200 patients [3]. Surgical resection is the only curative treatment, but is possible in only $20 \%$ of patients. The majority of patients present with advanced pancreatic cancer, namely locally advanced pancreatic cancer (LAPC) or metastatic pancreatic cancer (MPC). Advanced pancreatic cancer has a very poor prognosis, with a median overall survival of 3-8 months and a 5-year survival of $1-3 \%$ [2].

Gemcitabine monotherapy has been the standard palliative treatment for over a decade, providing a marginal survival benefit with a response rate of less than $10 \%$ [4]. Recently, the randomized trial of Conroy et al, comparing FOLFIRINOX (a combination of 5-fluorouracil, oxaliplatin, irinotecan and leucovorin) versus gemcitabine in metastatic disease, demonstrated a significant improvement in median overall survival (OS) of 11.1 versus 6.8 months ( $p<0.001)$, progression free survival (PFS) of 
6.4 versus 3.3 months $(p<0.001)$ as well as an increased response rate $(31.6 \%$ versus $9.4 \%)$ for FOLFIRINOX versus gemcitabine [5]. These results gave rise to the emergence of FOLFIRINOX as new standard care for MPC [6]. However, these improvements were at the expense of much higher toxicity rates, especially (febrile) neutropenia, thrombocytopenia, diarrhoea and sensory neuropathy and this has given rise to much concern [5].

Until recently, some of the physicians were reluctant to use FOLFIRINOX due to these high toxicity rates. This study reflects the experience in daily practice of a single academic centre with FOLFIRINOX by presenting the toxicity and efficacy of all patients with advanced pancreatic cancer treated with FOLFIRINOX.

\section{Methods}

\section{Patients}

A retrospective chart review was conducted of all patients with LAPC and MPC treated with FOLFIRINOX at the Academic Medical Center Amsterdam between April 2011 and January 2016. Patients who we treated with at least 1 cycle of FOLFIRINOX were included regardless of prior, second-line or additional treatment such as other chemotherapies, radiotherapy, surgery or local ablative therapies. In all cases, the unresectability (stage III and IV) was determined by the institution's multidisciplinary team. LAPC was defined as arterial involvement of $>90$ degrees and/or venous involvement of $>270$ degrees according to the current Dutch consensus laid down by the Dutch Pancreatic Cancer Group [7]. Administration of FOLFRINOX was started in April 2011 for MPC and from April 2012 for LAPC.

\section{Treatment}

Full dose FOLFIRINOX consisted of a 2 hour intravenous infusion of oxaliplatin $85 \mathrm{mg} / \mathrm{m}^{2}$, followed by a 2 hour intravenous infusion of leucovorin $400 \mathrm{mg} / \mathrm{m}^{2}$ concomitantly with 90 minutes of intravenous infusion of irinotecan $180 \mathrm{mg} / \mathrm{m}^{2}$, subsequently followed by 5 -fluorouracil (5-FU) 400 $\mathrm{mg} / \mathrm{m}^{2}$ as a bolus and $2.400 \mathrm{mg} / \mathrm{m}^{2}$ as a 46 hour continuous intravenous infusion. Dose modifications were made at the treating physician's discretion based on observed toxicity or on patient's request. Treatment was discontinued in case of unacceptable toxicity, progression of disease, pursuit of alternative therapies such as surgical resection or local ablative therapy or patient's request. All patients routinely received ondansteron and dexamethasone with each cycle for emesis prophylaxis.

\section{Data analysis}

Patients characteristics, dates of diagnosis, start of FOLFIRINOX treatment, dose modifications (modified or full dose at start, dose reductions during treatment, amount of cycles and discontinuations), all FOLFIRINOX related adverse events, maximum objective response rate (RR) and PFS were extracted from patient's medical records and radiology reports. Survival was extracted from the nationwide municipal registry, which records causes and dates of death for all inhabitants of the Netherlands. Moreover, prior, second-line and/or additional therapy was extracted if applicable. Adverse events were graded according to the National Cancer Institute Common Terminology Criteria for Adverse Events (CTCAE) Version 4.0 [8]. Radiological tumour response was defined according to the Response Evaluation Criteria in Solid Tumours (RECIST) on CT scans performed after 4 cycles of FOLFIRINOX and classified into 4 groups: complete response (disappearance of target lesion), partial response ( $\geq 30 \%$ decrease of diameter), stable disease (neither sufficient shrinkage nor sufficient increase) and progressive disease ( $\geq 20 \%$ increase in diameter). PFS was defined as the time elapsed from the start of FOLFIRINOX chemotherapy until the date of progression or death. OS was defined as the time elapsed from the start of FOLFIRINOX chemotherapy until death. Patients without events were censored at the last follow-up date.

\section{Statistics}

Quantitative data were described with the mean and standard deviation (SD) in case of normal distribution. In case of non-normally distribution, median and interquartile ranges (IQR) were reported. Categorical data were described by frequency distribution with the percentage. Continuous variables were compared using the Mann-Whitney $U$ test and categorical variables were compared using the Chi square test or Fisher's Exact test, depending of the number of groups. A p-value of 0,05 is considered to be statistically significant. OS and PFS analyses were estimated using the Kaplan-Meier method and were reported by the median and ranges. The median follow up duration was measured by the reverse Kaplan-Meier estimator [9]. All statistical analyses were performed with a statistical software package (SPSS, version 21.0, Armonk, NY: IBM Corp).

\section{Results}

\section{Characteristics of patients and tumours}

Between April 2011 and January 2016, 50 patients with LAPC $(n=18)$ and MPC $(n=32)$ were 
treated with FOLFIRINOX. All patients were treated with at least 1 cycle of FOLFIRINOX. The majority of patients $(68 \%)$ had a performance status (PS) of 0 according to the World Health Organisation (WHO). All other patients $(32 \%)$ had a WHO PS of 1 . Most tumours were located in the head of the pancreas $(60 \%)$ and in $44 \%$ of patients a biliary (covered metal) stent was placed before the start of chemotherapy.

Table 1. Patient and treatment characteristics

\begin{tabular}{|c|c|c|c|c|}
\hline Characteristic & $\begin{array}{l}\text { All } \\
\text { patients }\end{array}$ & $\begin{array}{l}\text { Locally } \\
\text { advanced }\end{array}$ & Metastatic & p-value $\$$ \\
\hline & $(n=50)$ & $(n=18)$ & $(n=32)$ & \\
\hline Age $^{*}$ & $55(49-66)$ & $61(48-67)$ & $55(50-61)$ & $0.46^{* *}$ \\
\hline Male & $30(60 \%)$ & $10(56 \%)$ & $20(63 \%)$ & $0.77^{\wedge}$ \\
\hline \multicolumn{5}{|l|}{ WHO PS } \\
\hline 0 & $34(68 \%)$ & $13(72 \%)$ & $21(66 \%)$ & \multirow[t]{2}{*}{$0.76^{\wedge}$} \\
\hline 1 & $16(32 \%)$ & $5(28 \%)$ & $11(34 \%)$ & \\
\hline \multicolumn{5}{|l|}{ Tumour location } \\
\hline Head & $30(60 \%)$ & $11(61 \%)$ & $19(59 \%)$ & \multirow[t]{4}{*}{$0.35^{\#}$} \\
\hline Uncinate & $12(24 \%)$ & $6(33 \%)$ & $6(19 \%)$ & \\
\hline Body & $5(10 \%)$ & $1(6 \%)$ & $4(13 \%)$ & \\
\hline Tail & $3(6 \%)$ & 0 & $3(9 \%)$ & \\
\hline Biliary stent & $22(44 \%)$ & $9(50 \%)$ & $13(41 \%)$ & $0.57^{\wedge}$ \\
\hline \multicolumn{5}{|l|}{ Prior chemotherapy regime } \\
\hline Gemcitabine first-line $^{+}$ & $9(18 \%)$ & $2(11 \%)$ & $7(22 \%)$ & \multirow[t]{2}{*}{$0.39^{\#}$} \\
\hline Gemcitabine adjuvant & $6(12 \%)$ & $1(6 \%)$ & $5(16 \%)$ & \\
\hline Prior radiotherapy & $1(2 \%)$ & $1(6 \%)$ & 0 & $0.36^{\wedge}$ \\
\hline \multicolumn{5}{|l|}{ Pre-treatment surgery } \\
\hline Bypass & $6(12 \%)$ & 0 & $6(19 \%)$ & \multirow[t]{2}{*}{$0.06^{\#}$} \\
\hline PPPD/Whipple & $6(12 \%)$ & $1(6 \%)$ & $5(16 \%)$ & \\
\hline Number of cycles & $8(5-9)$ & $8(6-9)$ & $8(4-9)$ & $0.40^{* *}$ \\
\hline \multicolumn{5}{|l|}{ Dose modifications } \\
\hline At start & $7(14 \%)$ & $3(17 \%)$ & $4(13 \%)$ & \multirow[t]{2}{*}{$0.69^{\wedge}$} \\
\hline After the first cycle & $38(76 \%)$ & $13(72 \%)$ & $25(78 \%)$ & \\
\hline \multicolumn{5}{|l|}{ Surgical treatment } \\
\hline $\begin{array}{l}\text { Exploration without } \\
\text { resection }\end{array}$ & $1(2 \%)$ & $1(6 \%)$ & 0 & \multirow[t]{4}{*}{$0.002^{\#}$} \\
\hline Resection & $1(2 \%)$ & $1(6 \%)$ & 0 & \\
\hline $\begin{array}{l}\text { Radiofrequency } \\
\text { ablation }\end{array}$ & $2(4 \%)$ & $2(11 \%)$ & 0 & \\
\hline $\begin{array}{l}\text { Irreversible } \\
\text { electroporation }\end{array}$ & $4(8 \%)$ & $4(22 \%)$ & 0 & \\
\hline \multicolumn{5}{|l|}{ Second line therapy } \\
\hline $\begin{array}{l}\text { Gemcitabine } \\
\text { monotherapy }\end{array}$ & $8(16 \%)$ & $5(28 \%)$ & $3(38 \%)$ & \multirow[t]{4}{*}{$0.27^{\#}$} \\
\hline Capecitabine & $1(2 \%)$ & 0 & $1(3 \%)$ & \\
\hline Gemcitabine based $^{++}$ & $5(10 \%)$ & 0 & $5(16 \%)$ & \\
\hline $\begin{array}{l}\text { Metformin plus } \\
\text { everolimus }\end{array}$ & $1(2 \%)$ & 0 & $1(3 \%)$ & \\
\hline
\end{tabular}

* at start of FOLFIRINOX treatment; \$ Comparing LAPC and MPC; **

Mann-Whitney U test; ^ Fisher's Exact test; \# Chi Square test; + Gemcitabine

monotherapy in 3 patients $(7 \%)$, combined with erlotinib in 3 patients $(7 \%)$, with mTOR-inhibitor in 2 patients $(4 \%)$ and with nab-paclitaxel in 1 patient $(2 \%),{ }^{++}$ gemcitabine combined with other chemotherapeutics or targeted agents bilirubin $(n=2)$, thrombopenia $(n=1)$, poor condition $(n=1)$ and on patient's request $(n=1)$. Most of the dose reductions were performed after the first cycle was administered (76\%). In the majority of cases the 5-FU bolus was modified: a reduction to $75 \%$ in 3 patients $(6 \%)$ and omitted in 34 patients $(68 \%)$. Treatment was stopped for progression of disease in 21 patients $(42 \%)$, for toxicity in 11 patients $(22 \%)$, ablative consolidation therapies in 4 patients $(8 \%)$, completion of the regimen (12 cycles) in 2 patients $(4 \%)$ and on patient's request in 9 patients $(18 \%)$. Three patients $(6 \%)$ were still treated with FOLFIRINOX at the time of analysing the data. Regardless of dose modifications, in the total group, patients were treated with a median number of 8 cycles (5-9).

The majority of patients $(82 \%)$ received FOLFIRINOX as first-line treatment for metastatic or locally advanced disease. Of these patients, 6 (15\%) had previously been resected and treated with adjuvant gemcitabine therapy. One patient developed a local recurrence and 5 showed distant metastases during follow up (Table 1). Second-line FOLFIRINOX therapy $(18 \%)$ was preceded by gemcitabine monotherapy or combined with other cytotoxic or targeted agents. One patient $(6 \%)$ was treated with gemcitabine combined with radiotherapy. Twelve patients underwent upfront surgery for the purpose of construction of a gastrojejunostomy, hepaticojejunostomy or both $(n=6)$ or resection with curative intent $(\mathrm{n}=6)$.

Table 2. Highest toxicity grade reported per patient during FOLFIRINOX

\begin{tabular}{lllll}
\hline & Grade 1 & Grade 2 & Grade 3 & Grade 4 \\
\hline Nausea & $23(46 \%)$ & $15(30 \%)$ & $1(2 \%)$ & 0 \\
Vomiting & $27(54 \%)$ & $7(14 \%)$ & $1(2 \%)$ & 0 \\
Neuropathy & $30(60 \%)$ & $4(8 \%)$ & 0 & 0 \\
Constipation & $13(26 \%)$ & $5(10 \%)$ & 0 & 0 \\
Diarrhoea & $24(48 \%)$ & $6(12 \%)$ & $2(4 \%)$ & 0 \\
Pain & $16(32 \%)$ & $5(10 \%)$ & 0 & $1(2 \%)$ \\
Alopecia & $12(24 \%)$ & $13(26 \%)$ & 0 & 0 \\
Hand-foot syndrome & $1(2 \%)$ & 0 & 0 & 0 \\
Mucositis & $7(14 \%)$ & 0 & 0 & 0 \\
Fatigue & $24(48 \%)$ & $16(32 \%)$ & $3(6 \%)$ & $1(2 \%)$ \\
Thrombocytopenia & $25(50 \%)$ & $5(10 \%)$ & $1(2 \%)$ & $1(2 \%)$ \\
Neutropenia & $3(6 \%)$ & $10(20 \%)$ & $10(20 \%)$ & $10(20 \%)$ \\
\hline
\end{tabular}

Grading according to CTCAE Version 4.0

\section{Tolerability}

Grade 3 and 4 toxicity occurred in 26 patients (52\%) (Table 3). Neutropenia was the most frequent grade $3 / 4$ toxicity $(40 \%)$, which led to dose reduction of FOLFIRINOX in all these patients and additionally to a delay in 6 patients and discontinuation in 4 patients. In the entire cohort, 4 patients $(8 \%)$ developed neutropenic fever. Other grade 3 and 4
$(14 \%)$ the regimen was modified already from the beginning, because of neutropenia $(n=2)$, high 
toxicities were nausea, vomiting, diarrhoea, fatigue, pain and thrombocytopenia. One patient (2\%) deceased from severe sepsis, during FOLFIRINOX treatment. The majority of grade 3 and 4 toxicities resolved after dose reduction (Figure 1). Only in case of thrombocytopenia, complications persisted in the same extent.

Table 3. Best response observed on CT-imaging after at least 4 cycles of FOLFIRINOX

\begin{tabular}{lll}
\hline & Locally advanced & Metastatic \\
\hline & $\left(\mathrm{n}=17^{*}\right)$ & $\left(\mathrm{n}=31^{*}\right)$ \\
Complete response & 0 & 0 \\
Partial response & $2(12 \%)$ & $10(32 \%)$ \\
Stable disease & $14(82 \%)$ & $16(52 \%)$ \\
Progressive disease & $1(6 \%)$ & $5(16 \%)$ \\
\hline * No radiological evaluation of 1 LAPC and 1 MPC patient
\end{tabular}

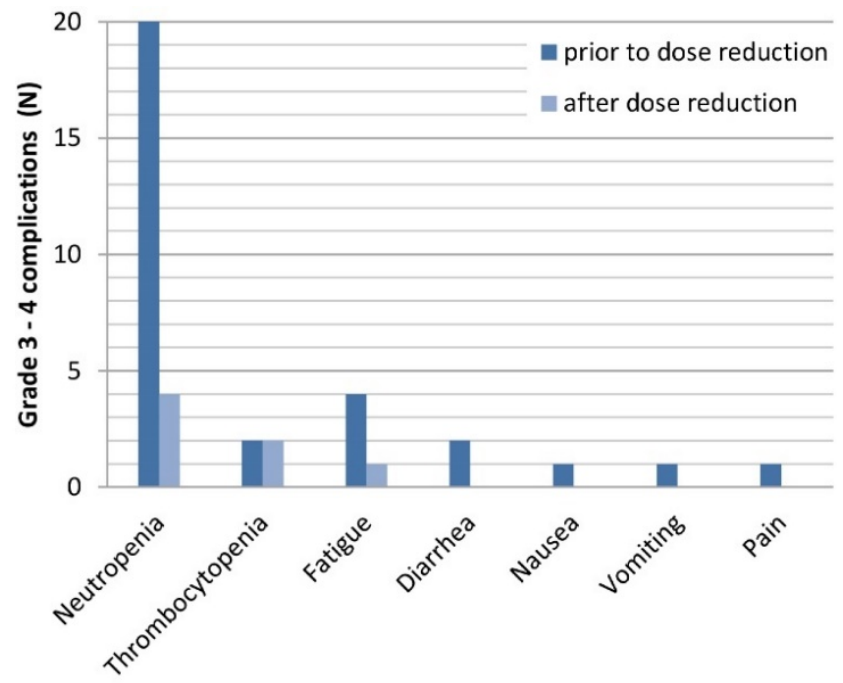

Figure 1. Each grade 3-4 complication prior and after dose reduction

\section{Response}

For most patients $(61 \%)$, the best response according to RECIST reported after at least 4 cycles of FOLFIRINOX was stable disease (Table 4). Two patients with LAPC and 10 patients with MPC showed partial response. None of the patients showed complete response. Seven patients had progression of disease at the first evaluation. Data on response were not available in two patients. Both patients completed only two cycles of FOLFIRINOX, one on patient's request and one died after severe sepsis. Thus, the response rate was $25 \%$ for the entire group, $12 \%$ for LAPC and $32 \%$ for MPC.

\section{Progression free and overall survival}

The median follow up period was 25.9 months (range 2.0 - 29.1 months). Survival data are represented in detail in Table 4 . The median overall survival for the entire group was 11.3 months (ranges 2.0-29.1 months). Patients who had partial response (19.6 months) had a significant higher median OS than patients with stable (11.2 months) or progressive disease (3.6 months) with a p- value of 0.012 and 0.001 , respectively. (Figure 2)

Patients with LAPC at start of FOLFIRINOX treatment had a median OS of 14.8 months (3.5-25.9 months) and a PFS of 10.3 months (1.8 - 25.9 months). In the MPC group the median OS was 9.0 months (2.0-29.1 months) with a PFS of 5.9 months (1.7 - 18.1 months).

Table 4. Survival and progression-free survival

\begin{tabular}{lllll}
\hline & All patients & \multicolumn{2}{l}{ Locally advanced Metastatic } & p-value $^{+}$ \\
\cline { 2 - 5 } & $(\mathrm{n}=50)$ & $(\mathrm{n}=18)$ & $(\mathrm{n}=32)$ & \\
\hline Survival & & & & \\
Overall & $11.2(2.0-29.1)$ & $14.8(3.5-25.9)$ & $9.0(2.0-29.1)$ & 0.154 \\
6-months & $78 \%$ & $94 \%$ & $67 \%$ & \\
1-year & $49 \%$ & $65 \%$ & $40 \%$ & \\
2-year & $8 \%$ & $10 \%$ & $5 \%$ & \\
PFS & & & & \\
Overall* & $7.3(1.7-25.9)$ & $10.3(1.8-25.9)$ & $5.9(1.7-18.1)$ & 0.005 \\
6-months & $64 \%$ & $89 \%$ & $50 \%$ & \\
1-year & $20 \%$ & $34 \%$ & $14 \%$ & \\
2-year & $3 \%$ & $8 \%$ & $0 \%$ & \\
\hline
\end{tabular}

\section{Consolidation therapy}

Surgical exploration was performed in $44 \%$ of the patients who initially presented with LAPC after at least 4 cycles of FOLFIRINOX. Six of those $(76 \%)$ were explored for the purpose of executing a local ablation (radiofrequency ablation or irreversible electroporation). One patient (6\%) had peritoneal metastases at exploration and one patient $(6 \%)$ underwent tumour resection. Pathology revealed an R1 resection. Following the pancreatoduodenectomy, this patient received another 4 cycles of FOLFIRINOX. The patient was alive after 13.5 months without signs of disease progression.

The other 6 patients (33\%) underwent local ablative therapy consisting of radiofrequency ablation $(n=2)$ and irreversible electroporation $(n=4)$. The median OS of these patients was 21.8 months (9.1-24.1 months) and PFS was 13.7 months (6.7-24.1 months). These patients received a median of 9 cycles (7-12 cycles) prior to ablation. Four of these patients received additional chemotherapy after the ablative procedure, consisting of FOLFIRINOX $(n=2)$ and gemcitabine monotherapy $(\mathrm{n}=2)$.

In the entire cohort, 13 patients $(26 \%)$ received second-line chemotherapy after progression under FOLFIRINOX treatment, consisting of gemcitabine monotherapy, or gemcitabine combined with other chemotherapeutics or targeted agents. 

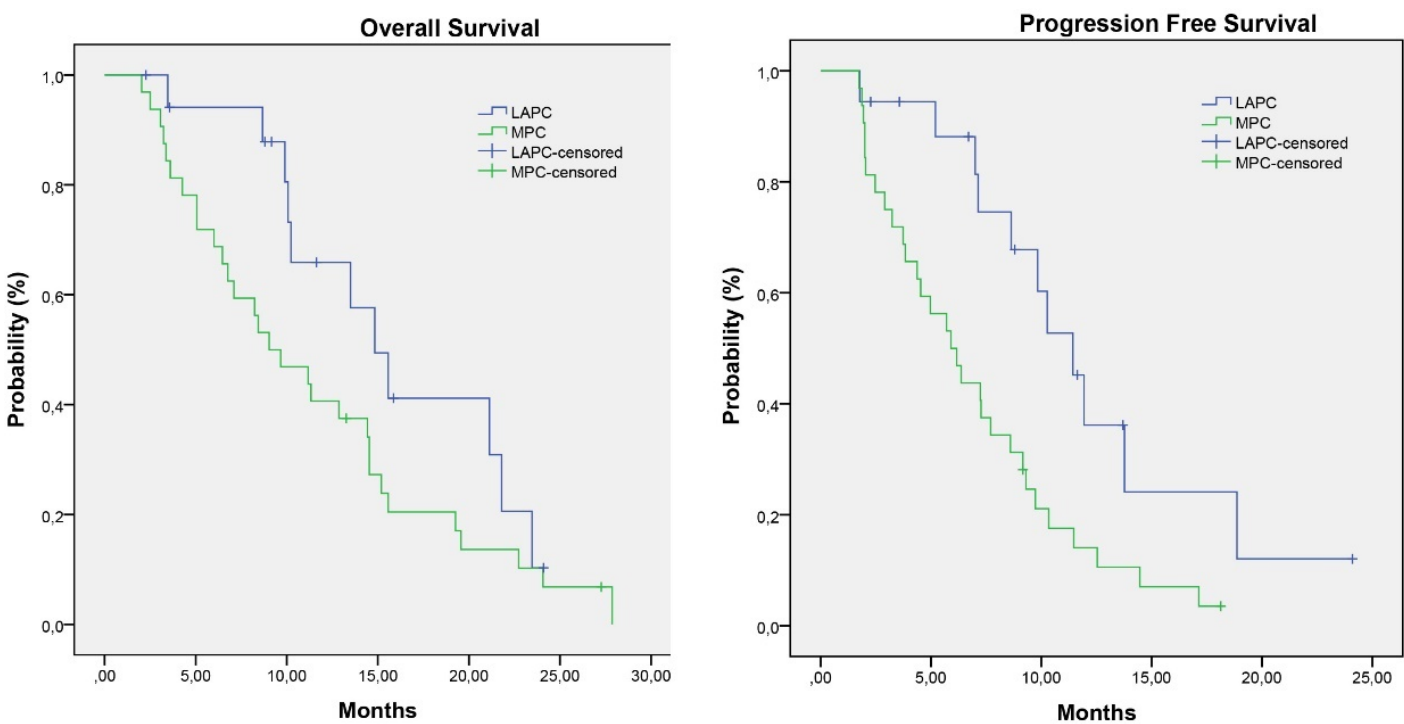

Figure 2. Kaplan-Meier survival estimates of overall survival and progression free survival

\section{Discussion}

In this retrospective cohort study of 50 patients with LAPC or MPC FOLFIRINOX was associated with acceptable toxicity, relatively high response rates and an overall 1- and 2-year survival rate of $65 \%$ and $10 \%$ in LAPC and $40 \%$ and $5 \%$ in MPC.

The vast majority of patients $(90 \%)$ were treated with a modified FOLFIRINOX regimen and a median number of 8 cycles (IQR 5-9). Nonetheless, the median OS rate of 14.8 months and 9.0 months, for LAPC and MPC respectively, were similar to the OS rates reported by studies with a lower percentage of dose modifications $(14 \%)$ and a higher median number of cycles $(10$ cycles $)[5,10]$. Moreover, the response rate of $26 \%$ seen in our patient population is comparable to previous reports of this regimen [5].

The high toxicity rates reported for FOLFIRINOX in previous publications has led to reluctance amongst clinicians in prescribing this regime. Our study, however, demonstrated that the majority of grade 3-4 complications related to FOLFIRINOX resolved by dose reduction (Figure 1). Our cohort furthermore suggests that, regardless of the dose reductions, patients can still benefit from FOLFIRINOX with survival rates comparable to the study of Conroy et al $[5,10]$.

A substantial portion of patients (36\%) had LAPC according to the Dutch definition (which may differ from definitions in other countries) [11]. It is notable that in our cohort, one-third of the LAPC patients was eligible for local ablative therapy. The median OS of these 6 patients was 21.8 months compared to 10.1 months in patients without ablative treatment $(p=0.04)$. One patient $(6 \%)$ was resected after FOLFIRINOX treatment. This percentage is lower than the resection rates reported in similar cohorts [12,13]. One of the main reasons is that surgical exploration after FOLFIRINOX was not routine practice at the time of this study. In contrast, the National Comprehensive Cancer Network (NCCN) defines 'unresectable' as arterial involvement beyond 180 degrees and unreconstructable venous involvement. Therefore, one can argue that more patients would have undergone resection if the less conservative guidelines of the NCCN were applied [14]. A recent study by Ferrone et al has suggested the necessity of surgical exploration of LAPC patients after FOLFIRINOX treatment, by demonstrating a R0 resection rate of $92 \%$ in 40 explored patients, who were initially assessed as being unresectable based on CT-imaging [15].

One of the limitations of this study is its retrospective design, which may lead to selection bias. A second limitation is the small number of patients.

Moreover, our cohort, like all previously published studies, only reflects the group of patients who actually received FOLFIRINOX and not the total group of patients with advanced pancreatic cancer and an appropriate WHO PS presented in our centre, including those who have not been treated with FOLFIRINOX the last years. Only such a cohort would give a complete representation of patients eligible for FOLFIRINOX. In April 2015, we started the multicenter randomized controlled PELICAN trial in the Netherlands in which we aim to include all patients with LAPC and collect all data throughout their treatment program [16]. In this way we can evaluate the indication, treatment and outcome in a complete cohort of patients with LAPC. 


\section{Conclusion}

Based on the outcomes of this study, FOLFIRINOX with convenient dose modifications shows relatively high response rates and a promising overall survival, with acceptable toxicity rates. Moreover, dose modifications, do not seem to effect the median overall survival, although more data are needed. This study supports the use of FOLFIRINOX as a treatment in LAPC and MPC in eligible patients.

\section{Competing Interests}

Professor doctor HWM van Laarhoven has served as a consultant for Celgene, Lilly, and Nordic, and has received unrestricted research funding from Bayer, Celgene, GSK, Janssen Cilag, Lilly, Merck Serono, MSD, Nordic, and Roche.

All of the other authors declare to have no conflict of interest or a source of funding relevant to this article.

\section{References}

1. [Internet] National Cancer Institute: Pancreatic Cancer Facts; Revised 5 February 2016. http://seer.cancer.gov/statfacts/html/pancreas.html

2. Ferlay J, Steliarova-Foucher E, Lortet-Tieulent J, et al. Cancer incidence and mortality patterns in Europe: estimates for 40 countries in 2012. Eur J Cancer. 2013 Apr; 49(6):1374-403.

3. [Internet] Oncoline: Pancreatic cancer; Revised 5 February 2016. http://www.oncoline.nl/pancreascarcinoom

4. Burris HA,3rd, Moore MJ, Andersen J, et al. Improvements in survival and clinical benefit with gemcitabine as first-line therapy for patients with advanced pancreas cancer: a randomized trial. J Clin Oncol. 1997; 15: 2403-13.

5. Conroy T, Desseigne F, Ychou M, et al. FOLFIRINOX versus gemcitabine for metastatic pancreatic cancer. N Engl J Med. 2011; 364: 1817-25.

6. Petrelli F, Coinu A, Borgonovo K, et al. FOLFIRINOX-based neoadjuvant therapy in borderline resectable or unresectable pancreatic cancer: a meta-analytical review of published studies. Pancreas. 2015; 44: 515-21.

7. [Internet] Definitions of resectability according to the Dutch Pancreatic Cancer Group; available at www.dpcg.nl and in the study protocol of the Pancreatic Locally advanced Irresectable Cancer Ablation in the Netherlands (PELICAN) trial, available at the Dutch Trial Register under number 5517. http://www.trialregister.nl/trialreg/admin/rctview.asp?TC=5517.

8. National Cancer Institute. Common Terminology Criteria for Adverse Events (CTCAE) version 4.0, May 2009

9. Schemper M, Smith TL. A note on quantifying follow-up in studies of failure time. Control Clin Trials. 1996; 17: 343-46

10. Rombouts SJ, Walma MS, Vogel JA, et al. Systematic Review of Resection Rates and Clinical Outcomes After FOLFIRINOX-Based Treatment in Patients with Locally Advanced Pancreatic Cancer. Ann Surg Oncol. 2016 Jul 1. [Epub ahead of print]

11. He J, Page AJ, Weiss M, et al. Management of borderline and locally advanced pancreatic cancer: where do we stand? World J Gastroenterol. 2014 Mar 7; 20(9): 2255-66.

12. Marthey L, Sa-Cunha A, Blanc JF, et al. FOLFIRINOX for locally advanced pancreatic adenocarcinoma: results of an AGEO multicenter prospective observational cohort. Ann Surg Oncol. 2015; 22: 295-301.

13. Sadot E, Doussot A, O'Reilly EM, et al. FOLFIRINOX Induction Therapy for Stage 3 Pancreatic Adenocarcinoma. Ann Surg Oncol. 2015; 22: 3512-21.

14. [Internet] National Comprehensive Cancer Network. NCCN guidelines version 2. 2015. Pancreatic Adenocarcinoma; 2015. http://www.tri-kobe.org/nccn/guideline/pancreas/english/pancreatic.pdf

15. Ferrone CR, Marchegiani G, Hong TS, et al. Radiological and surgical implications of neoadjuvant treatment with FOLFIRINOX for locally advanced and borderline resectable pancreatic cancer. Ann Surg. 2015; 261: $12-17$.

16. [Internet] Dutch Trial Register Number (NTR) 5517. http:/ / www.trialregister.nl/trialreg/admin/rctview.asp?TC $=5517$ 